\title{
Long non-coding RNA NEAT1/miR-320b/MSI2 axis regulates cisplatin resistance in ovarian cancer
}

\section{Type}

Research paper

\section{Keywords}

NEAT1, miR-320b, MSI2, cisplatin resistant, OC

\begin{abstract}
Introduction

Ovarian cancer $(\mathrm{OC})$ frequently occurs in postmenopausal women and it has higher mortality rate. Accumulating researches proved that long non-coding RNA nuclear paraspeckle assembly transcript 1 (NEAT1) involved in the progression of chemoresistance in human OC. Here, the study aimed to investigate the partial molecular mechanism of OC chemoresistance.
\end{abstract}

Material and methods

The levels of NEAT1 and microRNA-320b (miR-320b) were measured by qRT-PCR. Western blot was carried out to determine the protein levels that used in this research. Cell viability was identified via Cell Counting Kit-8 (CCK-8). Transwell assay was employed to determine migration and invasion. The relationship between miR-320b and NEAT1 or MSI2 was clarified by dual-luciferase reporter assay, RNA immunoprecipitation (RIP) and RNA pull down assay. Also, a murine xenograft assay was used to explore the effect of NEAT1 on cisplatin resistance in OC in vivo.

\section{Results}

The level of NEAT1 was significantly increased in cisplatin resistant OC cell lines. Downregulation of NEAT1 enhanced cisplatin sensibility in OVCAR-3/DDP and HEY/DDP cells. Furthermore, miR-320b was a target of NEAT1, and the effects of knockdown of NEAT1 on the cell viability, IC50 of cisplatin, migration and invasion in OVCAR-3/DDP and HEY/DDP were restored by the inhibitor of miR-320. In addition, miR-320b directly targeted MSI2 to regulate cisplatin sensibility in cisplatin resistant OC cells. In addition, downregulation of NEAT1 decreased cisplatin resistance in OC in vivo.

\section{Conclusions}

NEAT1 regulated cisplatin resistance through NEAT1/miR-320b/MSI2 axis in OC, which might offer a novel therapy target for the chemotherapy of OC. 
3

4 Chunling Zhao, Pingfen Zi, Degang Zhou*,

5 Department of Pharmacy, Eye, Ear, Nose and Larynx Hospital, Liaocheng People's

6 Hospital, Liaocheng, Shandong, China.

$7 \quad *$ Corresponding author: Degang Zhou. Department of Pharmacy, Eye, Ear, Nose and

8 Larynx Hospital, Liaocheng People's Hospital, No.Dongchang West Road 252000,

$9 \quad$ Liaocheng, Shandong, China.

Tel: $+86-0635-8278083$

Email: 405231097@qq.com

11

Running title: LncRNA NEAT1/miR-320b/MSI2 in ovarian cancer

列

(1)

.

15

16

.

18

19

\section{.}




\section{Abstract}

Introduction: Ovarian cancer (OC) frequently occurs in postmenopausal women and it has higher mortality rate. Accumulating researches proved that long non-coding RNA nuclear paraspeckle assembly transcript 1 (NEAT1) involved in the progression of chemoresistance in human OC. Here, the study aimed to investigate the partial molecular mechanism of OC chemoresistance.

Methods: The levels of NEAT1 and microRNA-320b (miR-320b) were measured by qRT-PCR. Western blot was carried out to determine the protein levels that used in this research. Cell viability was identified via Cell Counting Kit-8 (CCK-8). Transwell assay was employed to determine migration and invasion. The relationship between miR-320b and NEAT1 or MSI2 was clarified by dual-luciferase reporter assay, RNA immunoprecipitation (RIP) and RNA pull down assay. Also, a murine xenograft assay was used to explore the effect of NEAT1 on cisplatin resistance in OC in vivo.

Results: The level of NEAT1 was significantly increased in cisplatin resistant OC cell lines. Downregulation of NEAT1 enhanced cisplatin sensibility in OVCAR-3/DDP and HEY/DDP cells. Furthermore, miR-320b was a target of NEAT1, and the effects of knockdown of NEAT1 on the cell viability, IC50 of cisplatin, migration and invasion in OVCAR-3/DDP and HEY/DDP were restored by the inhibitor of miR-320. In addition, miR-320b directly targeted MSI2 to regulate cisplatin sensibility in cisplatin resistant OC cells. In addition, downregulation of NEAT1 decreased cisplatin resistance in OC in vivo.

Conclusion: NEAT1 regulated cisplatin resistance through NEAT1/miR-320b/MSI2 
axis in OC, which might offer a novel therapy target for the chemotherapy of OC.

Key words: NEAT1, miR-320b, MSI2, cisplatin resistant, OC

\section{Introduction}

Ovarian cancer (OC) is a common cause of cancer-related death in women. It frequently occurs in postmenopausal women, along with several months of abdominal pain and distension. Most patients come under observation have been in International Federation of Gynecology and Obstetrics [FIGO] stage III [1], usually therapy by using surgery and systemic treatment including chemotherapy, antiangiogenic drugs and PARP inhibitors and in selected cases radiotherapy. However, because of lack of effective chemotherapy strategy, the mortality of OC patients is still high, accompanied by a cure rate of only 30\% [2]. Exploring effective anti-cancer drugs or enhance chemosensitivity is of great significance. Oridonin was reported to repress OC cell metastasis [3], but whether it could be used for clinical treatment remains uncertain. Cisplatin (DDP) is a common and effective chemotherapy drug for the therapy of OC [4]. Thus this study focused on the regulatory mechanism of cisplatin (DDP) resistance. Over the past decades, studies indicated that chemoresistance was influenced by multiple factors, including long non-coding RNAs (lncRNAs) [5]. LncRNA is a kind of transcript, which has no function of translating into protein, but involved in the pathogenesis of various diseases, including but not limited to tumors. Accumulating researches proved that lncRNA modified cancer-associated processes, like metastasis and loss of imprinting [6]. Specially, upregulation of lncRNA anti-differentiation non- 
coding RNA (DANCR) involved in the advanced progression of tumor and poor prognosis in colorectal cancer [7], downregulation of it enhanced osteoblast differentiation by regulating enhancer of zeste homolog 2 (EZH2) [8]. What's more, IncRNAs was associated with the function and regulatory mechanism of OC [9]. Such as metastasis-associated lung adenocarcinoma transcript 1 (MALAT1) induced epithelial ovarian cancer cell proliferation and metastasis by phosphatidylinositol 3kinase/ protein kinase B (PI3K/AKT) signaling pathway [10]. Prostate cancerassociated transcript-1 (PCAT-1) was upregulated in OC and facilitated OC cell progression [11]. Myocardial infarction-associated transcript MIAT negative regulated miR-330-5p and acted as a tumor oncogenic lncRNA in epithelial ovarian cancer tumorigenesis [12]. Moreover, nuclear paraspeckle assembly transcript 1 (NEAT1) facilitated paclitaxel resistance in OC cells through microRNA-194/zincfinger ebox binding homeobox 1 (miR-194/ZEB1) axis [13]. All evidence suggested that NEAT1 participated in the progression and chemoresistance of OC, further researches for the molecular mechanism of the process needed to be conducted.

MicroRNAs (miRNAs) have been discovered to involve in the initiation and progression of tumors [14]. MicroRNA research in the context of tumor is a new strategy for pharmacologic approaches [15]. For example, microRNA-200, which was a family of tumor repressor miRNAs was strongly associated with inhibition of epithelial mesenchymal transformation, caused the inhibiting effect of cancer stem cells [16]. MicroRNA-179 targeted to PTEN-mediated PI3K/AKT signaling pathway to regulate proliferation and chemosensitivity of human ovarian cancer cells [17]. 
MicroRNA-331-3p reduced regulator of chromosome condensation 2 (RCC2) expression to impede OC cell proliferation and metastasis [18]. Previous research also shown that mircroRNA-320 acted as a hypoxia-regulated microRNA, was downregulated in hypoxic cells and regulated the expression of VEGF [15]. Meanwhile, miRNA also modulated the chemoresistance in human cancer [19]. MicroRNA-214 promoted cell survival and cisplatin resistance in OC by targeting phosphatase and tensin homolog deleted on chromosome ten (PTEN) [20]. MircroRNA-320b (miR-320b) as a target of X-inactive specific transcript (XIST), regulated the progression of osteosarcoma [21]. However, whether miR-320b modulates cisplatin resistance of human OC is unknown.

Musashi-2 (MSI2) has been proved to regulate the progression in amounts of human diseases [22]. For example, MSI2 contributed to cell metastasis by supporting transforming growth factor- $\beta$ (TGF- $\beta$ ) [23]. MSI2 was a predictive biomarker for liver metastasis and was related to poor prognosis in colorectal cancer [24]. Moreover, MSI2 induced the chemoresistance of liver cancer stem cells by regulating the activation of lin-28 homolog A (LIN28A) [25]. All evidence revealed that MSI2 may participate in chemoresistance in the therapy of human OC.

Herein, the research paid attention to the regulatory mechanism of NEAR1, miR320b and MSI2 in OC chemoresistance, and uncovered that NEAT1/miR-320b/MSI2 axis regulated the chemoresistance of $\mathrm{OC}$ in vivo and in vitro.

\section{Materials and methods}




\section{Cell culture}

Firstly, human OC cell lines OVCAR-3 and HEY were obtained from Chuan Qiu Biotechnology (Shanghai, China) and cultured in Roswell Park Memorial Institute (RPMI) medium. The cisplatin-resistent cell lines OVCAR-3/DDP and HEY/DDP were generated from their parental cell lines OVCAR-3 and HEY through treating with increasing concentration of cisplatin. All mediums were mixed with $10 \%$ fetal bovine serum (FBS, Gibco, Carlsbad, CA, USA) and $100 \mathrm{U} / \mathrm{mL}$ penicillin\&streptomycin (Gibco), in addition, OVCAR-3/DDP and HEY/DDP cells were added cisplatin (APExBIO Technology, Austin, TX, USA) with a final concentration of $2 \mathrm{ug} / \mathrm{mL}$. OC cells were incubated in $37^{\circ} \mathrm{C}$ with $5 \% \mathrm{CO}_{2}$.

\section{Cell Counting Kit-8 (CCK-8) assay}

CCK-8 assay was carried out for detection of cell viability and $\mathrm{IC}_{50}$ of cisplatin. Firstly, transfected cells were seeded in 96-well plates (Thermo Fisher Scientific, Waltham, MA, USA), and incubated for $48 \mathrm{~h}$, three repetitions were prepared for per sample. Subsequently, WST-8 (Sigma-Aldrich, Louis, MO, USA) was added into cells which was added different concentrations of cisplatin and cultured for another 2 h. Finally, microplate reader (Bio-Rad Laboratories, Philadelphia, PA, USA) was performed to measure OD value at $450 \mathrm{~nm}$. The cisplatin concentration repressed cell viability of $50 \%$ was considered cisplatin $\mathrm{IC}_{50}$ in vitro.

\section{Transwell assay}

Cell migration and invasion was determined by transwell assay. The upper chamber was coated without or with Matrigel (BD Biosciences, San Jose, CA, USA) for 
migration or invasion detection, respectively. Then, serum-free medium containing cells was added $\left(1 \times 10^{4}\right.$ cells for migration and $2 \times 10^{4}$ cells for invasion $)$. Otherwise, the basolateral chamber was added cell medium with 10\% serum. After incubation for $48 \mathrm{~h}$, the cells throughout the membrane was fixed by 4\% paraformaldehyde and dyed with $0.5 \%$ crystal violet solution. The cells were counted under a microscope (Thermo Fisher Scientific).

\section{Western blot assay}

OC cells were lysed by using Radio Immunoprecipitation Assay (RIPA) lysis buffer (Millipore, Bedford, MA, USA), and Extraction Buffer (Millipore) contained with protease inhibitors (Thermo Fisher Scientific) were applied to extract protein. Then protein concentration was examined by BCA Protein Assay Kit (Sangon Biotech, Shanghai, China). Subsequently, quantified protein which was denatured by boiling water was separated on SDS-polyacrylamide gel via electrophoresis and blotted onto polyvinylidene fluoride (PVDF) membranes (Millipore). Then membranes were blocked with $5 \%(\mathrm{w} / \mathrm{v})$ bovine serum albumin (Solarbio, Beijing, China) for $2 \mathrm{~h}$ at room temperature. Whereafter, PVDF membranes were incubated with primary antibodies which purchased from Abcam (Cambridge, MA, USA), including anti- Matrix metalloproteinases (MMP) 2(ab97779; 1:1000), anti-MMP9 (ab38898; 1:1000), antiMIS2 (ab73164; 1:1000) and anti-glyceraldehyde 3-phosphate dehydrogenase (GAPDH) (ab181602; 1:5000) overnight at $4^{\circ} \mathrm{C}$. Next, the membranes were incubated for $1 \mathrm{~h}$ at room temperature with secondary antibody (ab205718; 1:5000). Finally, protein signals were visualized utilizing Pierce ${ }^{\mathrm{TM}}$ ECL Western Blotting Substrate 
(Thermo Fisher Scientific) via Quantity One software (Bio-Rad Laboratories).

\section{Quantitative real-time polymerase chain reaction (qRT-PCR)}

The total RNA were extracted by Trizol (Invitrogen), and RNA was reverse transcribed into cDNA by using Thermo Scientific RevertAid First Strand cDNA Synthesis kit (Thermo Fisher Scientific). Then qRT-PCR was carried out to examine the expression of special RNA via SYBR Green real-time PCR kit (Takara, Dalian, China). In the end point, solution was reacted and signals were collected via ABI 7500 fast system (Applied Biosystems, Rockford, IL, USA). The levels of NEAT1, miR-320b and MSI2 were quantified via $2^{-\Delta \Delta \mathrm{Ct}}$ method and normalized by GAPDH (for NEAT1 and MSI2) and U6 (for miR-320b). Special primers were listed as below: NEAT1: (forward 5'CTT CCT CCC TTT AAC TTA TCC ATT CAC-3', reverse 5'-CTC TTC CTC CAC CAT TAC CAA CAA TAC-3'); MSI2: forward 5'-( and GAPDH: (forward 5'- ATT CCA TGG CAC CGT CAA GGC TGA -3', reverse 5'- TTC TCC ATG GTG GTG AAG ACG CCA -3'), which were synthesized in Ribobio, and The forward and reverse primers for miR-320b and U6 were purchased from Ribobio. The gene expression was presented using $2^{-\Delta \Delta C t}$ method.

\section{Transient transfection}

Special sequences, including Small interfering RNA (siRNA) against NEAT1 and siRNA negative control (si-control) miR-320 mimics (miR-320b), mimics blank control (miR-control), miR-320 inhibitor (miR-320 inhibitor), inhibitor negative control (inhibitor-control), overexpression vector of MSI2 (MSI2) and overexpression empty vector (pcDNA), bio-labeled probe of miR-320b (Bio-miR-320b), blank control 
177

(Bio-NC) and input (NC-Input, miR-320b-Input) as well as vectors of binding sites of wildtype (NEAT1 WT) and mutant (NEAT1 MUT) of NEAT1 and miR-320b, and 3'UTR sequences of wildtype (MSI2 3'UTR WT) and mutant (MSI2 3'UTR MUT) of MSI2 contained common fragments with miR-320b, short hairpin RNA (shRNA) of NEAT1 (sh-NEAT1) and shRNA negative control (sh-control) were synthesized in Ribobio (Guangzhou, China). Subsequently, vectors and oligonucleotide were transfected into OC cell lines by using Lipofectamine ${ }^{\mathrm{TM}} 2000$ (Invitrogen, Carlsbad, CA, USA) according to producer's manual, respectively.

\section{Dual-luciferase reporter assay}

LncBase Predicted v.2 was performed to predict the relationship between NEAT1 and miR-320b, and the interrelation between miR-320b and MSI2 was predicted utilizing Targetscan. Then vectors of binding sites of wildtype (NEAT1 WT) and mutant (NEAT1 MUT) of NEAT1 and miR-320b, and 3'-UTR sequences of wildtype (MSI2 3'UTR WT) and mutant (MSI2 3'UTR MUT) of MSI2 containing common fragments with miR-320b were synthesized in Ribibio. OVCAR-3/DDP and HEY/DDP cells were transfected with one of them and renilla plasmid, severally. Finally, luciferase activity was examined by Dual-Luciferase ${ }^{\circledR}$ Reporter Assay System (Promega, Madison, WI, USA) according to manual and analyzed via Varioskan Flash (Thermo Fisher Scientific).

\section{RNA immunoprecipitation (RIP) assay}

The assay was used EZ-Magna RIPTM RNA-Binding Protein Immunoprecipitation Kit (Millipore) to verify the relationship between NEAT1 and miR-320b. Firstly, OVCAR3/DDP and HEY/DDP cells were incubated for $48 \mathrm{~h}$ and lysed by using RIP-buffer. 
Then lysates were incubated with magnetic beads coated AGO2 (Argonaute-2) or IgG antibody which obtained from Abcam. Finally, residuum were washed off and RNA were harvested, qRT-PCR was carried to detect the enrichment of NEAT1 in OVCAR3/DDP and HEY/DDP cells.

\section{RNA pull down assay}

In the assay, bio-labeled probe of miR-320b (Bio-miR-320b), blank control (BioNC) and input (NC-Input, miR-320b-Input) were synthesized in Ribobio. Subsequently, OVCAR-3/DDP and HEY/DDP cells were transfected with probes, respectively, and incubated for $48 \mathrm{~h}$. Then, cells were lysed and lysates were incubated with StreptavidinDyna beads overnight at $4^{\circ} \mathrm{C}$ accompanied by RNA separation. Then the enrichment of NEAT1 was measured by qRT-PCR in vitro.

\section{Murine xenograft assay}

BALB/c nude mice (male, four-week-old) were purchased from Shanghai SLAC Laboratory Animal Co, Ltd. (Shanghai, China) and divided in three group ( $\mathrm{n}=6$ per group) randomly. The assay was performed according to guidelines of the National Animal Care and Ethics Institution and approved by Animal Research Committee of Eye, Ear, Nose and Larynx Hospital, Liaocheng People's Hospital. OVCAR-3/DDP cells were transfected with the lentivirus harboring sh-NEAT1 or negative control (shcontrol) constructed by Ribobio. Subsequently, stably transfected cells and OVCAR3/DDP cells were infected into mice subcutaneously, and then the mice were treated with DDP. The tumor volumes were examined after injected one week and then measured every three days until the mice were sacrificed at the $22 \mathrm{~d}$ post injection. The 
volumes were calculated following the formula: volume $\left(\mathrm{mm}^{3}\right)=$ width $^{2} \times$ length $/ 2$. Tumor samples were also took out and weighted after mice were sacrificed.

\section{Statistical analysis}

All the data were put forward as means \pm standard deviation (means $\pm \mathrm{SD}$ ) for three independent assays, Student's $t$ test was carried out to examine the difference between two groups. Additionally, one-way analysis of variance (ANOVA) was applied for multiple groups. Differences were considered significant statistically at $P$ less than 0.05 .

\section{Results}

\section{The level of NEAT1 was notably increased in cisplatin-resistant OC cell lines}

Firstly, cisplatin-resistant cell lines were established. The cell viability, $\mathrm{IC}_{50}$ of cisplatin, migration and invasion of the parental OC cells and cisplatin-resistant OC cells were detected to assess the cisplatin resistance. CCK-8 assay revealed that the cell viability and $\mathrm{IC}_{50}$ of cisplatin of OVCAR-3/DDP and HEY/DDP cells were higher than that of OVCAR-3 and HEY cells (Fig. 1A and 1B). Moreover, the capacities of migration and invasion of OVCAR-3/DDP $(P=0.0002, P<0.0001)$ and HEY/DDP cells $(P=0.0004$, $P<0.0001$ ) were both enhanced compared with their parental cells (Fig. 1C and 1D). Besides, the protein levels of migration-related proteins (MMP2 and MMP9) were also measured, and the results showed that MMP2 and MMP9 levels were significantly elevated in OVCAR-3/DDP and HEY/DDP cells $(P<0.0001)$ (Fig. 1E and 1F), which was consistent with the transwell result. These results indicated that cisplatin-resistant 
243

244

OC cell lines were successfully constructed. Then the level of NEAT1 were detected. The qRT-PCR result manifested that NEAT1 level was strikingly increased in cisplatin resistant OC cells (OVCAR-3/DDP and HEY/DDP cells) $(P<0.0001)$ (Fig. $1 \mathrm{G}$ and 1H). The aberrant expression of NEAT1 suggested that it might be involved in the regulation of cisplatin resistance in human OC.

\section{Knockdown of NEAT1 enhanced cisplatin sensitivity of cisplatin resistance OC} cell lines.

In order to investigate the potential role of NEAT1 in the regulatory mechanism of OC cisplatin resistance, si-NEAT1 or si-control was transfected into OVCAR-3/DDP and HEY/DDP cells, and the level of NEAT1 was significantly decreased in cisplatin resistant OC cells transfected with si-NEAT1 $(P=0.0013, P=0.0002)$ (Fig. 2A and 2B). Cell viability and cisplatin $\mathrm{IC}_{50}$ were notably reduced by si-NEAT1 in OVCAR3/DDP and HEY/DDP cells (Fig. 2C and 2D). Moreover, migration and invasion of OVCAR-3/DDP $(P=0.0001, P<0.0001)$ and HEY/DDP cells $(P<0.0002, P<0.0001)$ were also inhibited by NEAT1 knockdown (Fig. 2E and 2F). Furthermore, knockdown of NEAT1 downregulated the expression of MMP2 and MMP9 in vitro $(P<0.0001)$ (Fig. 2G and 2H). From the above, knockdown of NEAT1 increased cisplatin sensitivity of OVCAR-3/DDP and HEY/DDP cells.

\section{MiR-320b was a target gene of NEAT1.}

The binding sites between NEAT1 and miR-320b were predicted by LncBase Predicted 
v.2 (Fig. 3A). Dual-luciferase reporter assay was performed to clarify the interrelation between them, luciferase activity was evidently decreased in NEAT1 WT group $(P<$ 0.0001), while had no significant difference in NEAT1 MUT group $(P=0.6884, P=$ 0.9922), the evidence suggested that miR-320b was a target of NEAT1 (Fig. 3B and 3C). Moreover, the results of RIP $(\mathrm{P}<0.0001)$ and RNA pull down assay $(\mathrm{P}=0.0005$, $\mathrm{P}=0.0001$ ) were both verified the above conclusion (Fig. 3D-3G). In addition, the level of miR-320b was enhanced by sh-NEAT1 whereas repressed via overexpression of NEAT1 in OVCAR-3/DDP and HEY/DDP cells. These evidences revealed that NEAT1 directly targeted miR-320b, it might regulate OC cisplatin resistance by sponging miR$320 \mathrm{~b}$.

The effect of knockdown of NEAT1 on cisplatin sensitivity was reversed by miR320b inhibitor in cisplatin-resistant OC cell lines

In order to research regulatory mechanism between NEAT1 and miR-320b, si-control, si-NEAT1, si-NEAT1+miR-control or si-NEAT1+miR-320b was transfected into OVCAR-3/DDP and HEY/DDP cells, severally. The effect of si-NEAT1 on the expression of miR-320b was evidently reversed by miR-320b inhibitor $(P<0.0001)$ (Fig. 4A and 4B). The cell viability and $\mathrm{IC}_{50}$ of cisplatin which decreased by si-NEAT1 was regained by knockdown of miR-320b in SKOV3/DDP and A2780/DDP cells (Fig. 4C and 4D). What's more, si-NEAT1 repressed migration and invasion which was rescued by miR-320b inhibitor $(P<0.0001)$ (Fig. 4E and F). In addition, the downregulated MMP2 and MMP9 expression resulted by NEAT1 knockdown were 
restored by miR-320b inhibitor $(P<0.0001)$ (Fig. 4G and 4H). All evidences proved that the effect of NEAT1 knockdown on cisplatin sensitivity was reversed by miR-320b inhibitor in $\mathrm{OC}$ in vitro.

\section{MiR-320b directly targeted MSI2}

The interrelation between miR-320b and MSI2 was predicted via Targetscan and the binding sites were exhibited (Fig. 5A). Luciferase activity was significantly repressed by MIS2 3'UTR WT in OVCAR-3/DDP and HEY-3/DDP cell lines $(P<0.0001)$, while no notably change was observed in MSI2 3'UTR MUT group $(P=0.9706, P=0.9846)$

(Fig. 5B and 5C). Besides, the expression of MSI2 was distinctly decreased by miR320b mimics $(P<0.0001)$ (Fig. 5D). Knockdown of NEAT1 downregulated MSI2 expression, and this effect was reversed by miR-320b inhibitor (Fig. 5E and 5F). Additionally, the expression of MSI2 was markedly increased in OVCAR-3/DDP and HEY/DDP cells relative to VCAR-3 and HEY cells ( $<<0.0001)$ (Fig. 5G and 5H). In short, MSI2 was a target of miR-320b, and NEAT1 regulated MSI2 expression through sponging miR-320b.

\section{Overexpression of MSI2 restored the effect of miR-320b on cisplatin sensitivity in}

\section{cisplatin-resistant $\mathrm{OC}$ cell lines}

To further investigate the molecular mechanism between miR-320b and MSI2, OVCAR-3/DDP and HEY/DDP cells were transfected with miR-control, miR-320b, miR-320b+pcDNA or miR-320b+pcDNA-MSI2, respectively. The effect of miR-320b 
on MSI2 expression was rescued by pcDNA-MSI2 (Fig. 6A and 6B). In addition, the cell viability and cisplatin $\mathrm{IC}_{50}$ was decreased by $\mathrm{miR}-320 \mathrm{~b}$ while regained by overexpression of MSI2 in OVCAR-3/DDP and HEY/DDP cells (Fig. 6C and 6D). The effects of miR-320b mimics on migration and invasion were restored via cotransfecting with pcDNA-MSI2 in OVCAR-3/DDP and HEY/DDP cells (Fig. 6E and 6F). Moreover, overexpression of MSI2 recovered the effect of miR-320b mimics on MMP2 and MMP9 expression in cisplatin resistant OC cells (Fig. 6G and 6H). In brief, overexpress of MSI2 reversed the effect of miR-320b mimics on cell viability, cisplatin $\mathrm{IC}_{50}$, migration and invasion in cisplatin resistant $\mathrm{OC}$ cell lines, which meant that the effect of miR-320b mimics on increasing cisplatin sensibility was recovered by pcDNA-MSI2 in vitro.

\section{Knockdown of NEAT1 retarded the progression of OC tumors after cured with}

\section{DDP or not in vivo}

Nude mice were injected with OVCAR-3/DDP cells transfected with sh-control or shNEAT1 followed by treating with DDP. The tumor volumes and weight were both dramatically decreased in sh-NEAT1+DDP and sh-control+DDP groups, especially in sh-NEAT1+DDP group $(P<0.0001, P=0.0134)$ (Fig. 7A-7B). Meanwhile, the expression level of NEAT1 was significantly curbed while miR-320b expression was markedly enhanced via treatment with sh-NEAT1+DDP $(P=0.0003, P<0.0001)$ (Fig. 7C-7D). Finally, MSI2 expression was obviously decreased in sh-NEAT1+DDP group $(P=0.0001)$ (Fig. 7E). In conclusion, tumor progression was blocked via knockdown 
of NEAT1 in vivo.

\section{Discussion}

$\mathrm{OC}$ is a gynecologic malignancy which causing highly fatality ratio, and according to statistics, about $70 \%$ of patients will eventually succumb to their disease [26]. A large number of OC patients, with the exception of some well-differentiated early-stage cancer patients, undergo the treatment of chemotherapy with platinum (cisplatin or carboplatin) and paclitaxel [27]. The therapy shows promising effects at first, however, the occurrence and development of chemotherapy chemoresistance impose restrictions on successful treatment outcomes [28]. Therefore, chemoresistance is the primary problem in the treatment of ovarian cancer. The study used OVCAR-3/DDP and HEY/DDP cells as cisplatin resistant OC cell lines, and investigated the molecular mechanism of cisplatin resistance in OC.

Accumulating evidences have revealed that IncRNA, whose length over than 200 nucleotides, participated in the tumorigenesis of multiple cancers [29-31]. Specially, urothelial cancer associated 1 (UCA1) promoted breast cancer epithelial-mesenchymal transition by inducing Wnt/beta-catenin pathway [32]. Furthermore, IncRNA was also referred to human OC [33]. Human ovarian cancer-specific transcript 2 (HOST2) modulated cell biological behaviors by sponging microRNA let- $7 \mathrm{~b}$ in epithelial ovarian cancer [34]. NEAT1 accelerated metastasis of ovarian cancer via upregulating ROCK1 through miR-382-3p [35]. Also, NEAT1 could be regulated by p53 and was closely related with chemoresistance [36]. Previous studies revealed that NEAT1 enhanced 
cisplatin resistance in bladder cancer, nasopharyngeal carcinoma and anaplastic thyroid carcinoma [37-39]. On the contrary, NEAT1 reduced cisplatin resistance in lung cancer [40]. Besides, NEAT1 expression was consistent with the reactive oxygen species (ROS) level in cancer cells [41,42].The levels of ROS in drug resistant cancer cells are typically increased compared to non-multidrug resistance (MDR) cancer and normal cells [43]. Mounting evidence suggest that modulating cellular ROS levels can enhance MDR cancer cell death and sensitize MDR cancer cells to certain chemotherapeutic drugs $[44,45]$. These researches revealed that NEAT1 played different roles on cisplatin resistance in different cancers. Thus we explored the effect of NEAT1 on cisplatin resistance in OC. Our data uncovered that knockdown of NEAT1 augmented cisplatin sensibility in cisplatin resistant $\mathrm{OC}$ cells.

NEAT1 may exert its role through regulating downstream gene in the progress. For example, NEAT1 has been reported to regulate chemoresistance via miR-194/ZEB1, let-7a-5p/Rsf-1 and miR-9-5p/SPAG9 axis [10, 38, 39]. We also found that miR-194, let-7a-5p and miR-9-5p were upregulated, while ZEB1, Rsf-1 and SPAG9 were downregulated in cisplatin resistant OC cells (Fig. S1), suggesting that NEAT1 may response to cisplatin resistant through various pathways in OC cells. However, it still needs to be verified by further studies. In this study, we identified miR-320b as a target of NEAT1. A previous report proved that miR-320b was significantly downregulated in OC and might serve as biomarker for the prognosis of OC [45]. Yet there was no researches reported the effect of miR-320b on cisplatin resistance. Then this study showed that miR-320b was downregulated in cisplatin resistant OC cells, and miR- 
320b inhibitor reversed the effect of knockdown of NEAT1 on enhancing cisplatin sensibility in OC cells, implying the inhibitory role of miR-320b in cisplatin resistance. Additionally, further researches were also needed to investigate the regulatory mechanism of miR-320b in chemoresistance in cancers.

MSI2 has been suggested to participate in human haematopoietic stem cells via repressing aryl hydrocarbon receptor (AHR) signaling during the past decades [46]. It also contributed to cell migration and invasion of bladder cancer via activating Janus kinase 2/ signal transducer and activation of transcription 3 (JAK2/STAT3) pathway [47]. What's more, MSI2 was a novel regulator to modify paclitaxel sensitivity in OC cell lines [48]. In the research, MSI2 was a target by miR-320b, overexpression of it fortified cisplatin resistance in OVCAR-3/DDP and HEY/DDP cells. MSI2 may function as an oncogene, involve in chemoresistance in OC patients.

In conclusion, the level of NEAT1 was upregulated in cisplatin-resistant OC cells, subsequent assay showed that it regulated cisplatin resistance by NEAT1/miR320b/MSI2 axis. However, the chemotherapy resistance of various tumors still needs to be further studied in the future.

\section{Conclusion}

The level of NEAT1 was notably increased in cisplatin resistant OC cell lines. Knockdown of NEAT1 significantly augmented the cisplatin sensibility in OVCAR3/DDP and HEY/DDP cells. What's more, miR-320b as a target gene of NEAT1, miR$320 \mathrm{~b}$ inhibitor reversed the effect of knockdown of NEAT1 on the cell viability, $\mathrm{IC}_{50}$ of cisplatin, migration and invasion in OVCAR-3/DDP and HEY/DDP cells. In addition, 
397 MSI2 was directly targeted by miR-320b. MiR-320b mimics significantly decreased 398 cell viability, cisplatin $\mathrm{IC}_{50}$ migration and invasion in OVCAR-3/DDP and HEY/DDP 399 cells, while these effects were rescued by MSI2 overexpression vector. Furthermore, 400 downregulation of NEAT1 curbed the progression of tumor in vivo. In brief, NEAT1 401 regulated cisplatin resistant through NEAT1/miR-320b/MSI2 axis in human cisplatin 402 resistant OC.

403

404 Acknowledgements

405 Not applicable.

406

407 Funding

408 No funding was received.

409

410 Availability of data and materials

411 The analyzed data sets generated during the present study are available from the 412 corresponding author on reasonable request.

Ethics approval and consent to participate

The present study was approved by the ethical review committee of Eye, Ear, Nose and 


\section{Patient consent for publication}

$420 \quad$ Not applicable.

421

422 Competing interests

423 The authors declare that they have no competing interests.

424

425 References

426 1. Jayson G C, Kohn E C, Kitchener H C, Ledermann J A. Ovarian cancer. Lancet 2014; 384: 1376-88.

428

429

2. Lengyel E. Ovarian cancer development and metastasis. Am J Pathol 2010; 177:

430

431

432

433

434

435

3. Wang Y, Zhu Z. Oridonin inhibits metastasis of human ovarian cancer cells by suppressing the mTOR pathway. Arch Med Sci 2019; 15(4): 1017-1027.

4. Jayson GC, Kohn EC, Kitchener HC, Ledermann JA. Ovarian cancer. Lancet 2014; 384(9951): 1376-1388.

5. Yang G, Lu X, Yuan L. LncRNA: a link between RNA and cancer. Biochim Biophys Acta 2014; 1839: 1097-109.

6. Gibb E A, Brown C J, Lam W L. The functional role of long non-coding RNA in human carcinomas. Mol cancer 2011; 10: 38.

7. Liu Y, Zhang M, Liang L, Li J, Chen Y-X. Over-expression of IncRNA DANCR is associated with advanced tumor progression and poor prognosis in patients with colorectal cancer. Int J Clin Exp Pathol 2015; 8: 11480. 
441

442

443

444

445

446

447

448

449

450

451

452

453

454

455

456

457

458

459

460

461

462

8. Zhu L, Xu P-C. Downregulated LncRNA-ANCR promotes osteoblast differentiation by targeting EZH2 and regulating Runx2 expression. Biochem Biophy Res Commun 2013; 432: 612-7.

9. Ren C, Li X, Wang T, Wang G, Zhao C, Liang T, et al. Functions and mechanisms of long noncoding RNAs in ovarian cancer. Int J Gynecol Cancer 2015; 25: 5669.

10. Jin Y, Feng S, Qiu S, Shao N, Zheng J. LncRNA MALAT1 promotes proliferation and metastasis in epithelial ovarian cancer via the PI3K-AKT pathway. Eur Rev Med Pharmacol Sci 2017; 21: 3176-84.

11. Gu LP, Jin S, Xu RC, et al. Long non-coding RNA PCAT-1 promotes tumor progression by inhibiting miR-129-5p in human ovarian cancer. Arch Med Sci $2019 ; 15(2): 513-521$.

12. Shao S, Tian J, Zhang H, Wang S. LncRNA myocardial infarction-associated transcript promotes cell proliferation and inhibits cell apoptosis by targeting miR330-5p in epithelial ovarian cancer cells. Arch Med Sci 2018; 14(6): 1263-1270.

13. An J, Lv W, Zhang Y. LncRNA NEAT1 contributes to paclitaxel resistance of ovarian cancer cells by regulating ZEB1 expression via miR-194. Oncotarget Ther 2017; 10: 5377 .

14. Melo S A, Kalluri R. Molecular pathways: microRNAs as cancer therapeutics. Clin Cancer Res 2012; 18: 4234-9.

15. Kulshreshtha R, Davuluri RV, Calin GA, Ivan M. A microRNA component of the hypoxic response. Cell Death Differ 2008;15(4):667-71. 
16. Feng X, Wang Z, Fillmore R, Xi Y. MiR-200, a new star miRNA in human cancer. Cancer lett 2014; 344: 166-73.

17. Zhihong Z, Rubin C, Liping L, et al. MicroRNA-1179 regulates proliferation and chemosensitivity of human ovarian cancer cells by targeting the PTEN-mediated PI3K/AKT signaling pathway. Arch Med Sci 2019; 16(4): 907-914.

18. Buranjiang G, Kuerban R, Abuduwanke A, Li X, Kuerban G. MicroRNA-331-3p inhibits proliferation and metastasis of ovarian cancer by targeting RCC2. Arch Med Sci. 2019;15(6):1520 - 1529.

19. Sorrentino A, Liu C-G, Addario A, Peschle C, Scambia G, Ferlini C. Role of microRNAs in drug-resistant ovarian cancer cells. Gynecol Oncol 2008; 111: 47886.

20. Yang H, Kong W, He L, Zhao J-J, O'Donnell J D, Wang J, et al. MicroRNA expression profiling in human ovarian cancer: miR-214 induces cell survival and cisplatin resistance by targeting PTEN. Cancer Res 2008; 68: 425-33.

21. Lv G-Y, Miao J, Zhang X-L. Long noncoding RNA XIST promotes osteosarcoma progression by targeting Ras-related protein RAP2B via miR-320b. Oncol Res 2018; 26: 837-46.

22. Barbouti A, Höglund M, Johansson B, Lassen C, Nilsson P-G, Hagemeijer A, et al. A novel gene, MSI2, encoding a putative RNA-binding protein is recurrently rearranged at disease progression of chronic myeloid leukemia and forms a fusion gene with HOXA9 as a result of the cryptic $t(7 ; 17)(p 15 ;$ q23). Cancer Res 2003; 63: $1202-6$. 
23. Kudinov A E, Deneka A, Nikonova A S, Beck T N, Ahn Y-H, Liu X, et al. Musashi2 (MSI2) supports TGF- $\beta$ signaling and inhibits claudins to promote non-small cell lung cancer (NSCLC) metastasis. Proc Natl Acad Sci U S A 2016; 113: 695560.

24. Zong Z, Zhou T, Rao L, Jiang Z, Li Y, Hou Z, et al. Musashi2 as a novel predictive biomarker for liver metastasis and poor prognosis in colorectal cancer. Cancer Med 2016; 5: 623-30.

25. Fang T, Lv H, Wu F, Wang C, Li T, Lv G, et al. Musashi 2 contributes to the stemness and chemoresistance of liver cancer stem cells via LIN28A activation. Cancer lett 2017; 384: 50-9.

26. Jemal A, Siegel R, Ward E, Hao Y, Xu J, Thun M J. Cancer statistics, 2009. CA Cancer J Clin 2009; 59: 225-49.

27. Harries M, Gore M. Part I: chemotherapy for epithelial ovarian cancer-treatment at first diagnosis. The lancet oncology 2002; 3: 529-36.

28. Eltabbakh G H, Awtrey C S. Current treatment for ovarian cancer. Expert Opin Pharmacother 2001; 2: 109-24.

29. Zhang H, Chen Z, Wang X, Huang Z, He Z, Chen Y. Long non-coding RNA: a new player in cancer. J hematol oncol 2013; 6: 37.

30. Wang K C, Chang H Y. Molecular mechanisms of long noncoding RNAs. Mol cell 2011; 43: 904-14.

31. Fatica A, Bozzoni I. Long non-coding RNAs: new players in cell differentiation and development. Nat Rev Genet 2014; 15: 7. 
32. Xiao $\mathrm{C}, \mathrm{Wu} \mathrm{C}, \mathrm{Hu} \mathrm{H}$. LncRNA UCA1 promotes epithelial-mesenchymal transition (EMT) of breast cancer cells via enhancing Wnt/beta-catenin signaling pathway. Eur Rev Med Pharmacol Sci 2016; 20: 2819-24.

33. Nikpayam E, Tasharrofi B, Sarrafzadeh S, Ghafouri-Fard S. The role of long noncoding RNAs in ovarian cancer. Iran Biomed J 2017; 21: 3.

34. Gao Y, Meng H, Liu S, Hu J, Zhang Y, Jiao T, et al. LncRNA-HOST2 regulates cell biological behaviors in epithelial ovarian cancer through a mechanism involving microRNA let-7b. Hum Mol Genet 2014; 24: 841-52.

35. Liu Y, Wang Y, Fu X, Lu Z. Long non-coding RNA NEAT1 promoted ovarian cancer cells' metastasis through regulation of miR-382-3p/ROCK1 axial. Cancer Sci 2018;109(7):2188- 2198.

36. Adriaens C, Standaert L, Barra J, Latil M, Verfaillie A, Kalev P, et al. p53 induces formation of NEAT1 lncRNA-containing paraspeckles that modulate replication stress response and chemosensitivity. Nat Med 2016; 22: 861.

37. Zhao W, Li W, Jin X, Niu T, Cao Y, Zhou P, et al. Silencing long non-coding RNA NEAT1 enhances the suppression of cell growth, invasion, and apoptosis of bladder cancer cells under cisplatin chemotherapy. Int J Clin Exp Pathol 2019;12(2):549-558.

38. Liu F, Tai Y, Ma J. LncRNA NEAT1/let-7a-5p axis regulates the cisplatin resistance in nasopharyngeal carcinoma by targeting Rsf-1 and modulating the Ras-MAPK pathway. Cancer Biol Ther 2018;19(6):534-542.

39. Yan P, Su Z, Zhang Z, Gao T. LncRNA NEAT1 enhances the resistance of anaplastic 

expression. Int J Oncol 2019;55(5):988-002.

40. Jiang P, Wu X, Wang X, Huang W, Feng Q. NEAT1 upregulates EGCG-induced CTR1 to enhance cisplatin sensitivity in lung cancer cells. Oncotarget 2016;7(28):43337 - 43351 .

41. Gast M, Rauch BH, Haghikia A, et al. Long noncoding RNA NEAT1 modulates immune cell functions and is suppressed in early onset myocardial infarction patients. Cardiovasc Res 2019; 115(13): 1886-1906.

42. Chen A, Jiang P, Zeb F, et al. EGCG regulates CTR1 expression through its prooxidative property in non-small-cell lung cancer cells [published online ahead of print, 2020 Jan 14]. J Cell Physiol. 2020;10.1002/jcp.29451.

43. Cui Q, Wang JQ, Assaraf YG, et al. Modulating ROS to overcome multidrug resistance in cancer. Drug Resist Updat 2018; 41: 1-25.

44. Wang H, Gao Z, Liu X, et al. Targeted production of reactive oxygen species in mitochondria to overcome cancer drug resistance. Nat Commun 2018; 9(1): 562.

45. Cha SY, Choi YH, Hwang S, Jeong JY, An HJ. Clinical Impact of microRNAs Associated With Cancer Stem Cells as a Prognostic Factor in Ovarian Carcinoma. J Cancer. 2017;8(17):3538 - 3547. Published 2017 Sep 30. doi:10.7150/jca.20348

46. Rentas S, Holzapfel N T, Belew M S, Pratt G A, Voisin V, Wilhelm B T, et al. Musashi-2 attenuates AHR signalling to expand human haematopoietic stem cells. Nature 2016; 532: 508.

47. Yang C, Zhang W, Wang L, Kazobinka G, Han X, Li B, et al. Musashi-2 promotes 

pathway. Lab Invest 2016; 96: 950.

48. Lee J, An S, Choi Y M, Lee J, Ahn K J, Lee J H, et al. Musashi-2 is a novel regulator of paclitaxel sensitivity in ovarian cancer cells. Int J Oncol 2016; 49: 1945-52.

556

557

Figure legends

Fig. 1 The level of NEAT1 was notably increased in cisplatin resistant OC cell lines.

(A and B) The cell viability and $\mathrm{IC}_{50}$ of cisplatin in OVCAR-3, OVCAR-3/DDP, HEY, and HEY/DDP cells were assessed by CCK-8 assay. (C and D) Migration and invasion of OVCAR-3, OVCAR-3/DDP, HEY, and HEY/DDP cells were determined by transwell assay. (E and F) The protein levels of MMP2 and MMP9 were detected by 
western blot. ( $\mathrm{G}$ and $\mathrm{H}$ ) The expression of NEAT1 was detected in OVCAR-3, OVCAR-3/DDP, HEY, and HEY/DDP cells by qRT-PCR. Date are represented as means $\pm \mathrm{SD} ; * P<0.05 * * P<0.01, * * * P<0.001, * * * * P<0.0001$

Fig. 2 Knockdown of NEAT1 enhanced cisplatin sensitivity of cisplatin resistance OC cell lines.

OVCAR-3/DDP and HEY/DDP cells were transfected with si-NEAT1 or si-control, respectively. (A and B) The level of NEAT1 was determined by qRT-PCR. (C and D) CCK-8 asssay was carried out to detect cell viability and $\mathrm{IC}_{50}$ of cisplatin. (E and F) Migration and invasion were determined by transwell assay ( $\mathrm{G}$ and $\mathrm{H})$ The protein levels of MMP2 and MMP9 were detected by western blot. Date are represented as means $\pm \mathrm{SD} ; * * P<0.01, * * * P<0.001, * * * * P<0.0001$

Fig. 3 MiR-320b was a target gene of NEAT1.

(A) The relationship between NEAT1 and miR-320b was predicted by LncBase Predicted v.2. (B and C) NEAT1 WT and NEAT1 MUT was transfected into OVCAR3/DDP and HEY/DDP cells, severally, and luciferase activity was determined by dualluciferase reporter assay in vitro. (D-G) RIP assay and RNA pull down assay were preformed to verify the interrelation between NEAT1 and miR-320b in SKOV3/DDP and A2780/DDP cells. Date are represented as means $\pm \mathrm{SD} ; * * * P<0.001, * * * * P<$ 0.0001 .

Fig. 4 The effect of knockdown of NEAT1 on cisplatin sensitivity was reversed by 
595

596

597 598

599

600

601

602

603

604

605

606

607

608

609

610

611

612

613

614

615

616

miR-320b in cisplatin resistance OC cell lines.

SKOV3/DDP and A2780/DDP cells were transfected with si-control, si-NEAT1, siNEAT1+miR-control or si-NEAT1+miR-320b, respectively. (A and B) The level of miR-320b was measured by qRT-PCR. (C and D) CCK-8 asssay was carried out to detect cell viability and IC50 of cisplatin. (E and F) Migration and invasion were determined by transwell assay ( $\mathrm{G}$ and $\mathrm{H}$ ) The protein levels of MMP2 and MMP9 were detected by western blot. Date are represented as means $\pm \mathrm{SD} ; * * P<0.01, * * * P<$ $0.001, * * * * P<0.0001$

\section{Fig. 5 MiR-320b directly targeted MSI2.}

(A) Targetscan was used to predict the relationship between miR-320b and MSI2. (B and C) Luciferase activity was measured utilizing dual-luciferase reporter assay.

The expression of MSI2 in OVCAR-3/DDP and HEY/DDP cells transfected with miRcontrol or miR-320b was determined by western blot, severally. (E and F) The expression of MSI2 in OVCAR-3/DDP and HEY/DDP cells transfected with si-control, si-NEAT1, si-NEAT1+miR-control or si-NEAT1+miR-320b was determined by western blot, severally. ( $\mathrm{G}$ and $\mathrm{H}$ ) expression of MSI2 in OVCAR-3, OVCAR-3/DDP, HEY, and HEY/DDP cells was determined by western blot. Date are represented as means $\pm \mathrm{SD} ; * * * P<0.001, * * * * P<0.0001$

Fig. 6 Overexpression of MSI2 restored the effect of miR-320b on cisplatin sensitivity in cisplatin resistance $\mathrm{OC}$ cell lines. 
617

618

619

620

621

622

623

624

625

626

627

628

629

630

631

632

633

634

635

636

637

638

MiR-control, miR-320b, miR-320b+pcDNA or miR-320b+MSI2 were transfected into

OVCAR-3/DDP and HEY/DDP cells, respectively. (A and B) The expression of MSI2 were measured by and western blot. (C and D) CCK-8 asssay was carried out to detect cell viability and IC50 of cisplatin. (E and F) Migration and invasion were determined

by transwell assay ( $\mathrm{G}$ and $\mathrm{H}$ ) The protein levels of MMP2 and MMP9 were detected by western blot. Date are represented as means $\pm \mathrm{SD} ; * * P<0.01, * * * P<0.001, * * * * P$ $<0.0001$.

Fig. 7 Knockdown of NEAT1 retarded the progression of OC tumors after cured with cisplatin or not in vivo.

OVCAR-3/DDP cells transfected with sh-control or sh-NEAT1 were injected nude mice subcutaneously. (A) Tumor volumes were measured every three days after injection for one week. (B) Tumor weights were also measured after mice were sacrificed. (C and D) QRT-PCT was carried out to determine the levels of NEAT1 and miR-320b. (E) The expression of MSI2 was analyzed western blot. Date are represented as means $\pm \mathrm{SD}$; $* P<0.05, * * * P<0.001, * * * * P<0.0001$.

Fig. S1 MiR-194, let-7a-5p and miR-9-5p were upregulated, while ZEB1, Rsf-1 and SPAG9 were downregulated in cisplatin resistant $\mathrm{OC}$ cells. (A and B) The expression of miR-194, let-7a-5p and miR-9-5p in OVCAR-3, OVCAR-3/DDP, HEY, and HEY/DDP cells was detected by qRT-PCR. (C and D) The mRNA levels of ZEB1, Rsf-1 and SPAG9 were in OVCAR-3, OVCAR-3/DDP, HEY, and HEY/DDP cells was 
639 detected by qRT-PCR. ${ }^{*} P<0.05$. 
A
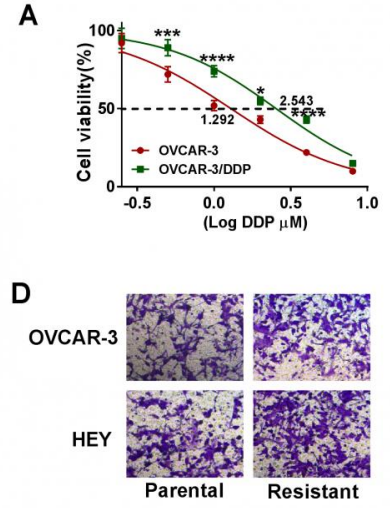

$\mathbf{F}$

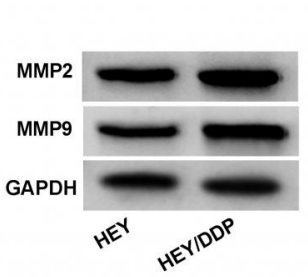

B
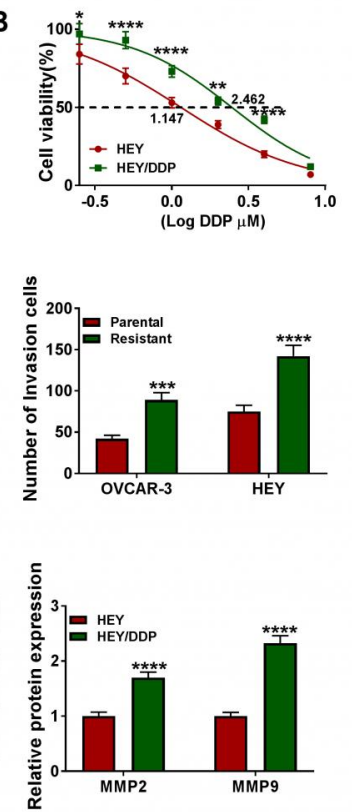

C
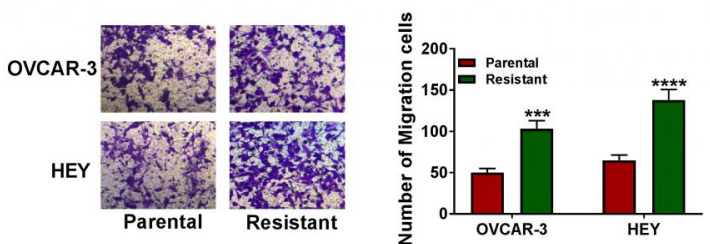

E
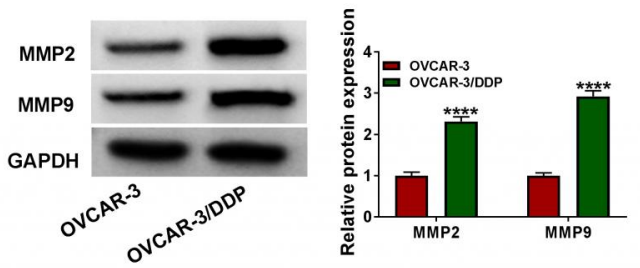

G

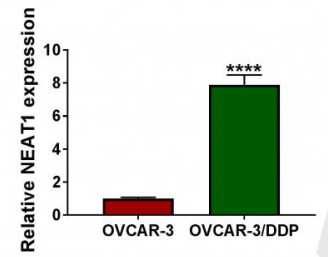

$\mathbf{H}$

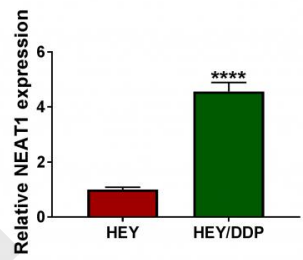

Fig. 1 The level of NEAT1 was notably increased in cisplatin resistant OC cell lines. (A and B) The cell viability and IC50 of cisplatin in OVCAR-3, OVCAR-3/DDP, HEY, and HEY/DDP cells were assessed by CCK-8 assay. ( $C$ and $D)$ Migration and invasion of OVCAR-3, OVCAR-3/DDP, HEY, and HEY/DDP cells were determined by transwell assay. ( $E$ and $F$ ) The protein levels of MMP2 and MMP9 were detected by western blot. ( $G$ and $H$ ) The expression of NEAT1 was detected in OVCAR-3, OVCAR-3/DDP, HEY, and HEY/DDP cells by $q R T-P C R$. Date are represented as means $\pm S D \square{ }^{*} P<0.05{ }^{* *} P<0.01,{ }^{* * *} P<0.001$, ${ }^{* * * *} \mathrm{P}<0.0001$. 
A

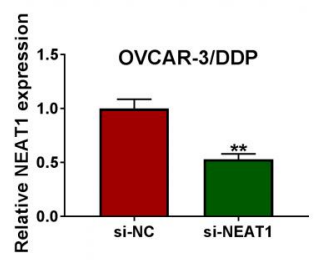

E

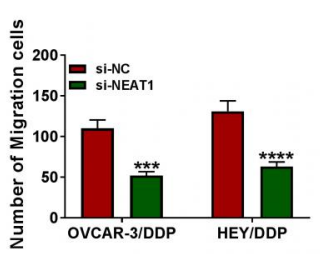

H

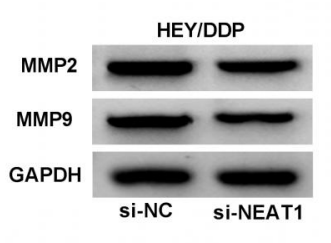

B

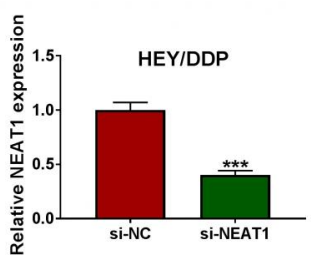

$\mathbf{F}$

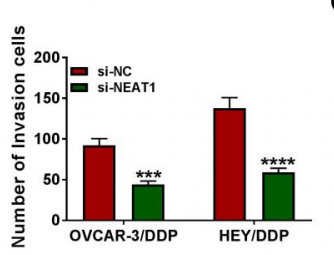

C
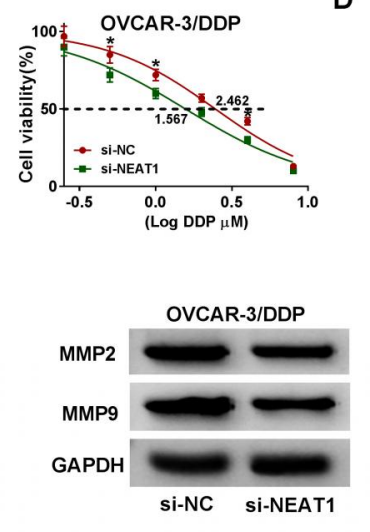

D
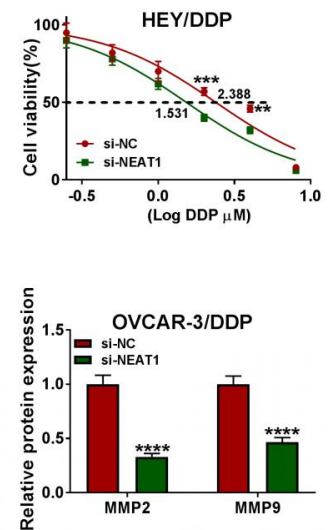

Fig. 2 Knockdown of NEAT1 enhanced cisplatin sensitivity of cisplatin resistance OC cell lines.

OVCAR-3/DDP and HEY/DDP cells were transfected with si-NEAT1 or si-control, respectively. ( $A$ and $B$ ) The level of NEAT1 was determined by qRT-PCR. (C and D) CCK-8 asssay was carried out to detect cell viability and IC50 of cisplatin. (E and F) Migration and invasion were determined by transwell assay ( $G$ and $H)$ The protein levels of MMP2 and MMP9 were detected by western blot. Date are represented as means $\pm S D{ }^{* *} P<0.01$, ${ }^{* * *} \mathrm{P}<0.001,{ }^{* * * *} \mathrm{P}<0.0001$ 


\section{A}

NEAT1 WT 5' uggCCUUUUGAAAAAAGCAGCUUUa 3'

miR-320b 3' aaCGGGAGA----GUUGGGUCGAAAa 5'

NEAT1 MUT 5' ugAUGUAUCGAAAGgAGACUACGGa 3'

B

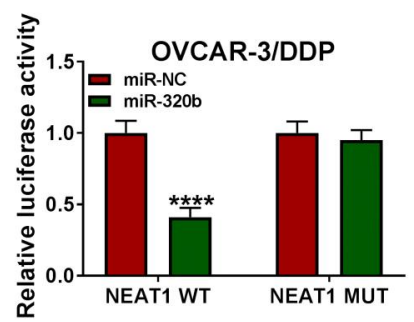

$\mathbf{E}$

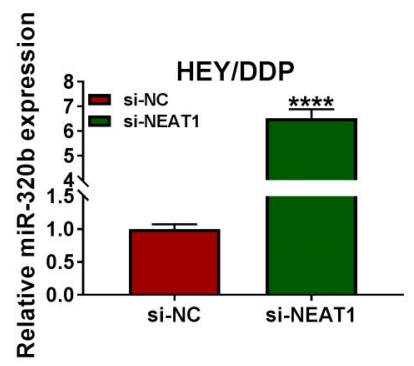

C

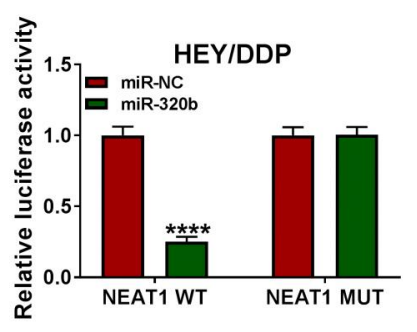

$\mathbf{F}$

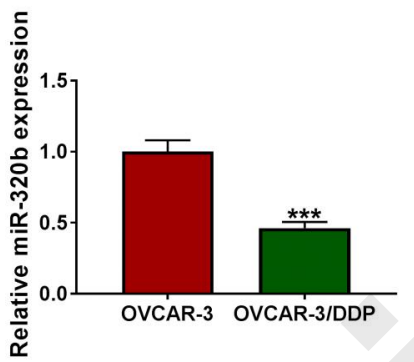

D

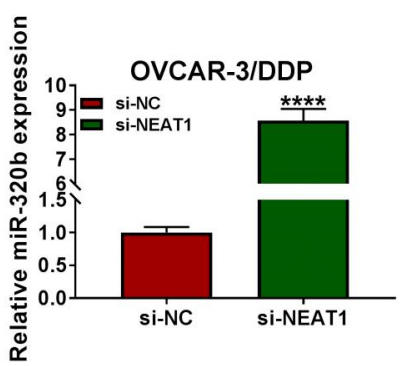

G

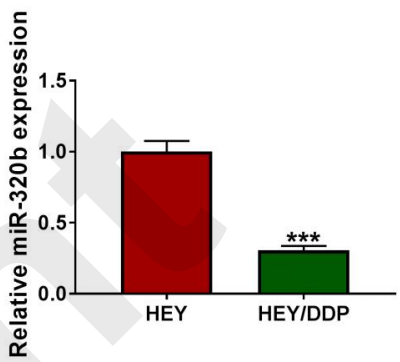

Fig. 3 MiR-320b was a target gene of NEAT1.

(A) The relationship between NEAT1 and miR-320b was predicted by LncBase Predicted v.2. (B and C) NEAT1 WT and NEAT1 MUT was transfected into OVCAR-3/DDP and HEY/DDP cells, severally, and luciferase activity was determined by dual-luciferase reporter assay in vitro. (D-G) RIP assay and RNA pull down assay were preformed to verify the interrelation between NEAT1 and miR-320b in SKOV3/DDP and A2780/DDP cells. Date are represented as means $\pm \mathrm{SD} \square^{* * *} \mathrm{P}<0.001,{ }^{* * * *} \mathrm{P}<0.0001$. 

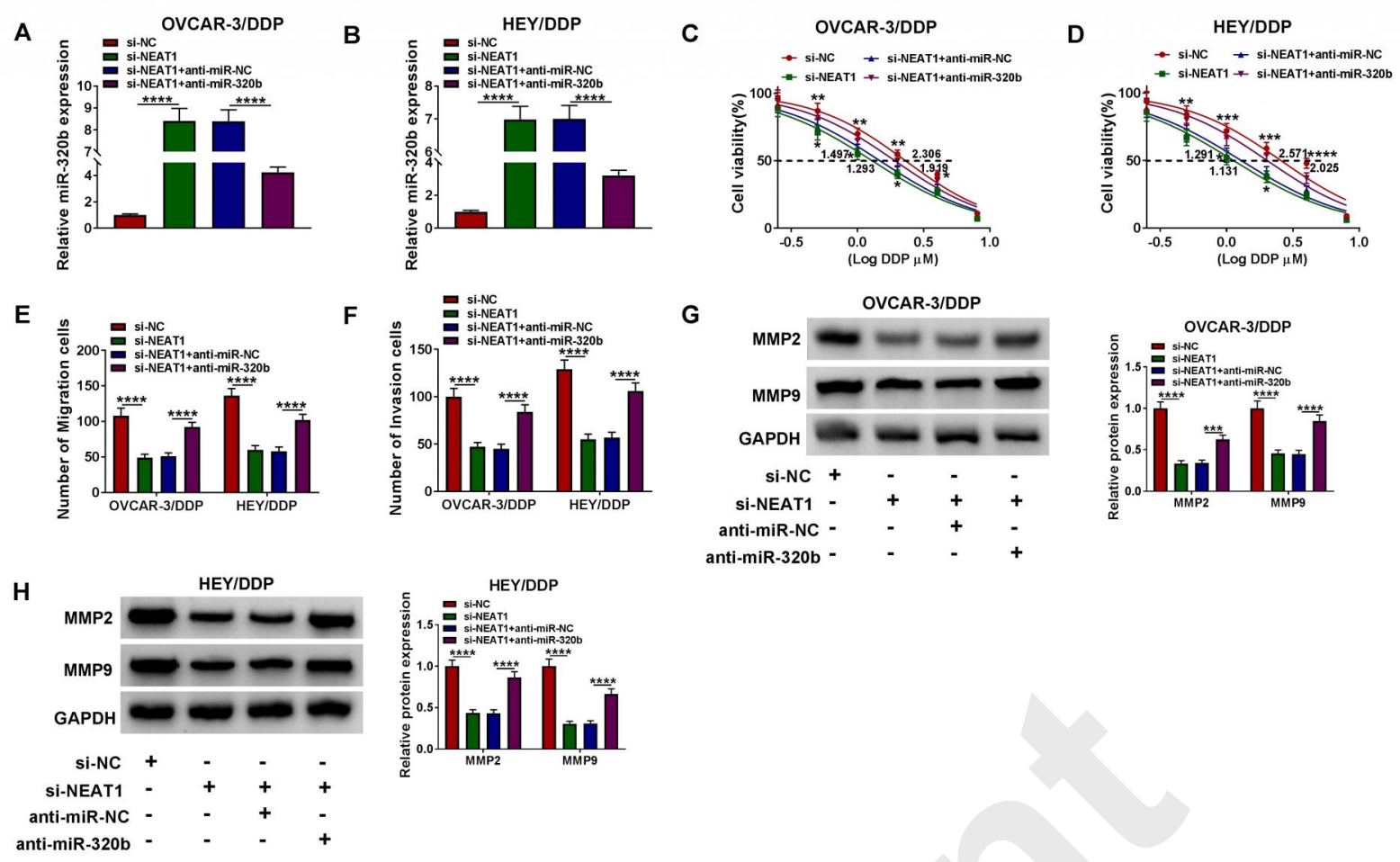

Fig. 4 The effect of knockdown of NEAT1 on cisplatin sensitivity was reversed by miR-320b in cisplatin resistance $\mathrm{OC}$ cell lines.

SKOV3/DDP and A2780/DDP cells were transfected with si-control, si-NEAT1, siNEAT1+miR-control or si-NEAT1+miR-320b, respectively. (A and B) The level of miR-320b was measured by qRT-PCR. (C and D) CCK-8 asssay was carried out to detect cell viability and IC50 of cisplatin. ( $E$ and $F$ ) Migration and invasion were determined by transwell assay $(\mathrm{G}$ and $\mathrm{H})$ The protein levels of MMP2 and MMP9 were detected by western blot. Date are represented as means $\pm \mathrm{SD} \square{ }^{* *} \mathrm{P}<0.01,{ }^{* * *} \mathrm{P}<0.001$, ${ }^{* * * *} \mathrm{P}<0.0001$. 

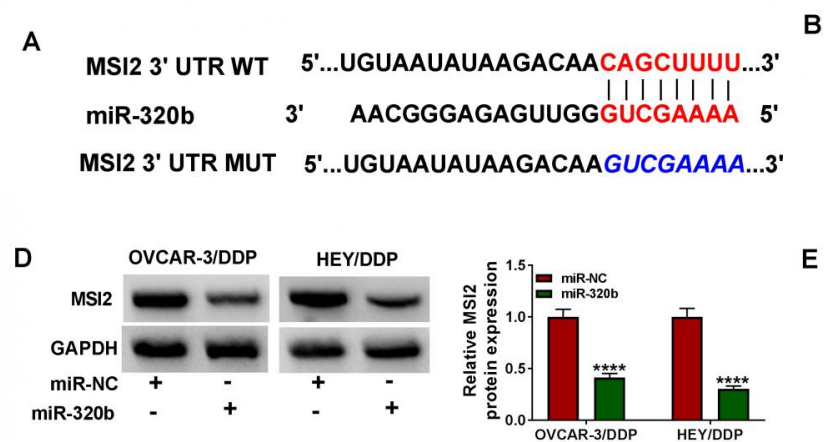

F

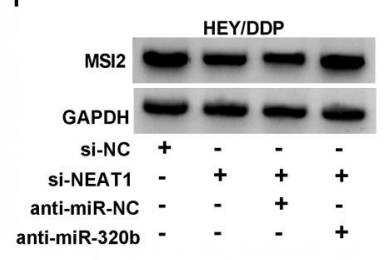

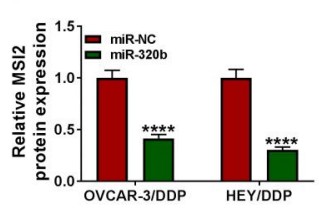

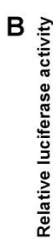

E

G $\mathrm{MSI2}$

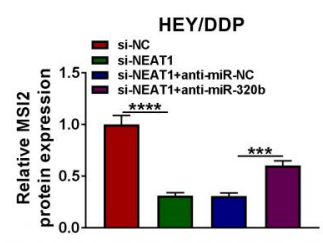

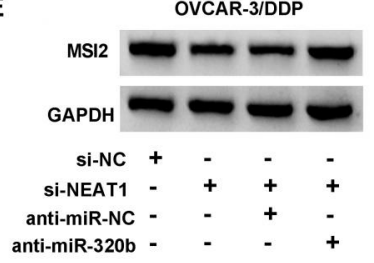

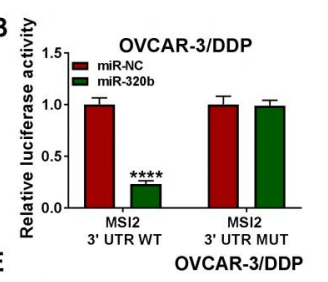

C
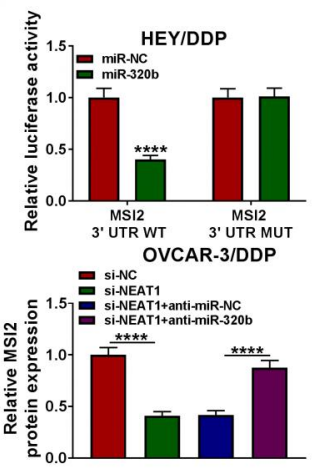

H

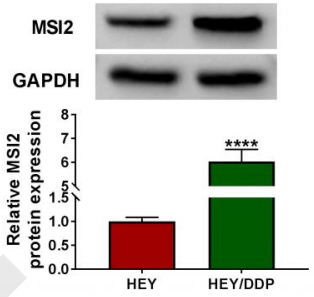

Fig. 5 MiR-320b directly targeted MSI2.

(A) Targetscan was used to predict the relationship between miR-320b and MSI2. (B and C) Luciferase activity was measured utilizing dual-luciferase reporter assay. (D) The expression of MSI2 in OVCAR-3/DDP and HEY/DDP cells transfected with miR-control or miR-320b was determined by western blot, severally. ( $E$ and $F$ ) The expression of MSI2 in OVCAR-3/DDP and HEY/DDP cells transfected with si-control, si-NEAT1, si-NEAT1+miRcontrol or si-NEAT1+miR-320b was determined by western blot, severally. ( $G$ and $H$ ) expression of MSI2 in OVCAR-3, OVCAR-3/DDP, HEY, and HEY/DDP cells was determined by western blot. Date are represented as means $\pm S D \square{ }^{* * *} P<0.001,{ }^{* * * *} P<$ 0.0001 . 
A

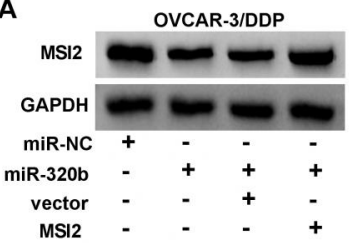

C OVCAR-3/DDP

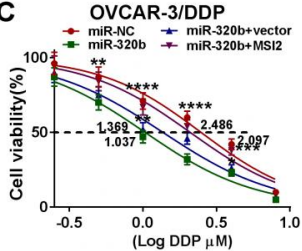

G

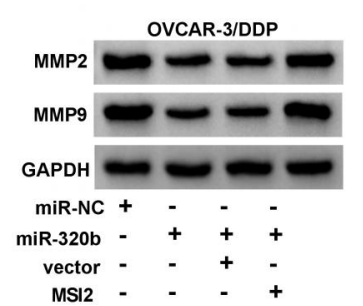

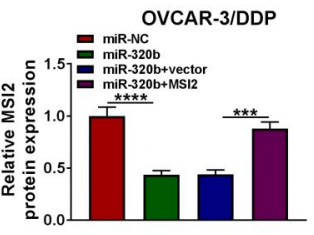

D
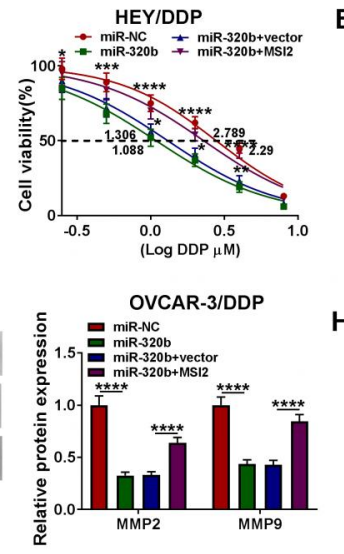

B
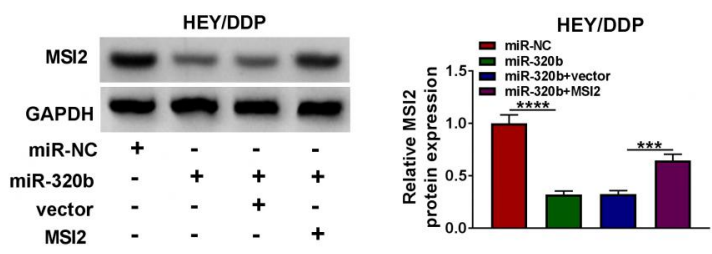

$E$

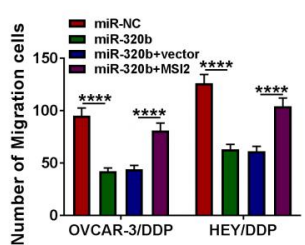

$\mathbf{F}$

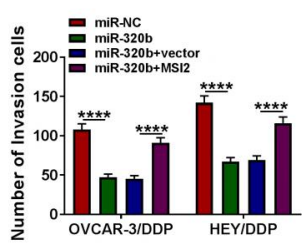

H

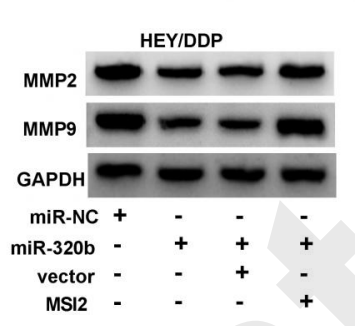

Fig. 6 Overexpression of MSI2 restored the effect of miR-320b on cisplatin sensitivity in cisplatin resistance OC cell lines.

MiR-control, miR-320b, miR-320b+pcDNA or miR-320b+MSI2 were transfected into OVCAR-3/DDP and HEY/DDP cells, respectively. (A and B) The expression of MSI2 were measured by and western blot. (C and D) CCK-8 asssay was carried out to detect cell viability and IC50 of cisplatin. ( $E$ and F) Migration and invasion were determined by transwell assay $(\mathrm{G}$ and $\mathrm{H}$ ) The protein levels of MMP2 and MMP9 were detected by western blot. Date are represented as means $\pm \mathrm{SD} \square{ }^{* *} \mathrm{P}<0.01$, ${ }^{* * *} \mathrm{P}<0.001$, ${ }^{* * *} \mathrm{P}<$ 0.0001 . 
A

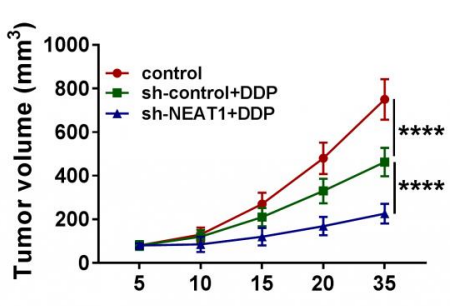

D

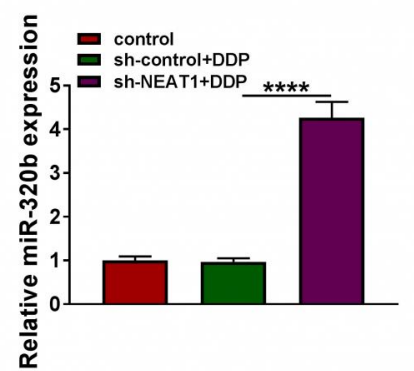

B

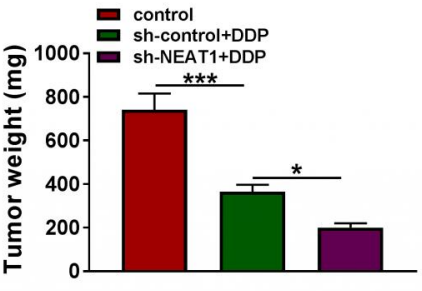

E

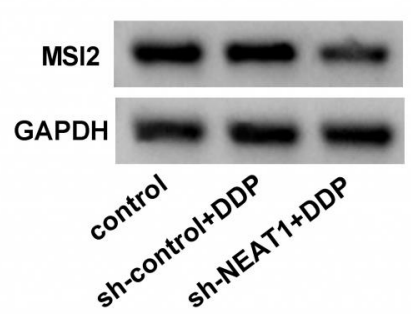

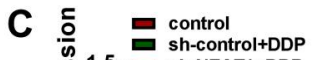
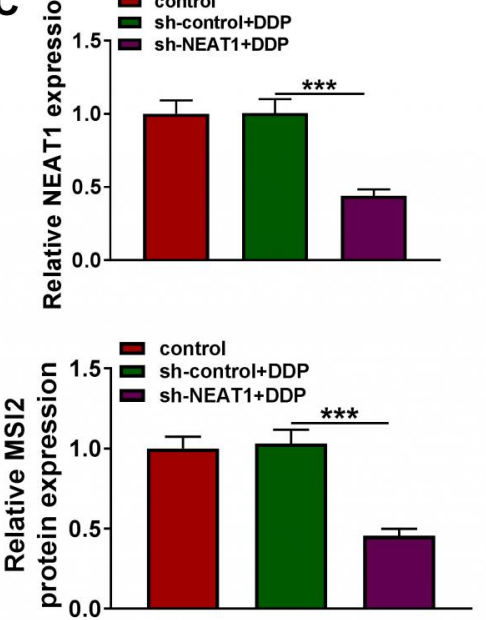

Fig. 7 Knockdown of NEAT1 retarded the progression of OC tumors after cured with cisplatin or not in vivo.

OVCAR-3/DDP cells transfected with sh-control or sh-NEAT1 were injected nude mice subcutaneously. (A) Tumor volumes were measured every three days after injection for one week. (B) Tumor weights were also measured after mice were sacrificed. (C and D) QRTPCT was carried out to determine the levels of NEAT1 and miR-320b. (E) The expression of MSI2 was analyzed western blot. Date are represented as means $\pm S D \square{ }^{*} P<0.05,{ }^{* * *} P<$ $0.001,{ }^{* * * *} \mathrm{P}<0.0001$. 

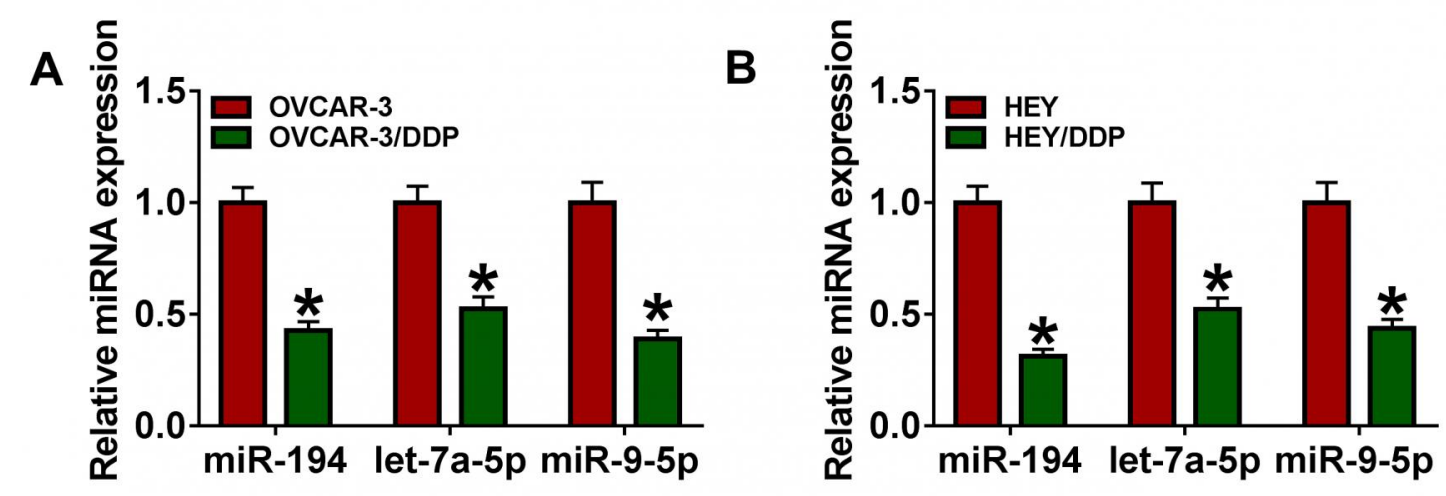

C

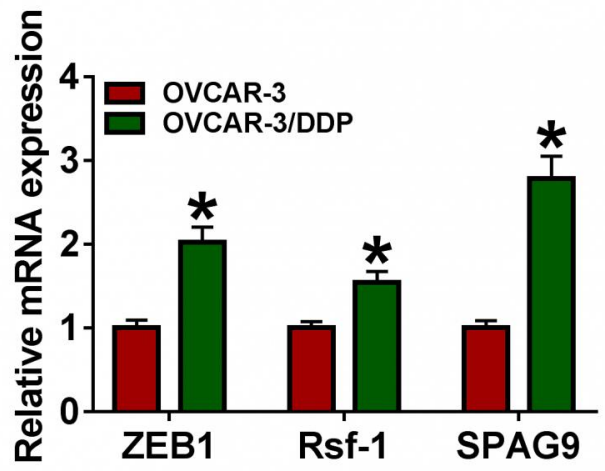

D

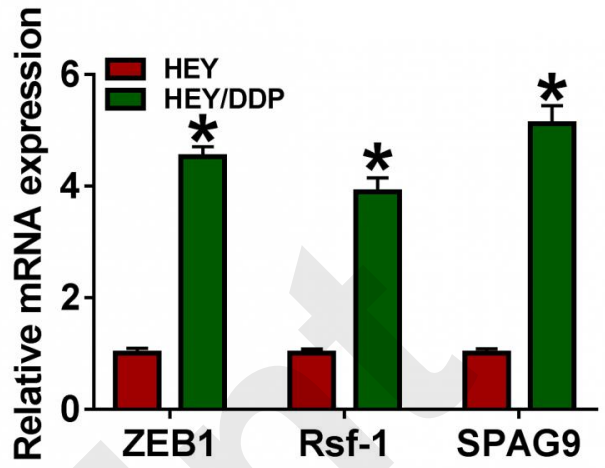

Fig. S1 MiR-194, let-7a-5p and miR-9-5p were upregulated, while ZEB1, Rsf-1 and SPAG9 were downregulated in cisplatin resistant OC cells. (A and B) The expression of miR-194, let-7a-5p and miR-9-5p in OVCAR-3, OVCAR-3/DDP, HEY, and HEY/DDP cells was detected by qRT-PCR. (C and D) The mRNA levels of ZEB1, Rsf-1 and SPAG9 were in OVCAR-3, OVCAR-3/DDP, HEY, and HEY/DDP cells was detected by qRT-PCR. ${ }^{*} P<0.05$. 